\title{
CORRIGENDUM: MATHEMATIKA VOLUME 15, 1968
}

\section{J. W. THOMAS}

Irrotational gravity waves of finite height: a numerical study. The sentence on page 146 in which equation (30) occurs should read:

The method consists of expanding the equation as

$$
u(t)=\mu \sin \left[\frac{1}{3 \pi} \int_{0}^{\pi} \frac{u(s)}{1+\int_{0}^{s} u(y) d y} L_{i}(s, t) d s\right]=\mu T_{i} u(t)
$$

$i=1,2$, where $u(t)=\mu \sin \theta(t)$. 